\title{
Evaluation of patellar tendinopathy using the single leg decline squat test: Is pain location important?
}

\begin{abstract}
Study Design A cross-sectional study of non-elite volleyball players aged 13-17years.

Objectives To evaluate the presence and location of pain during the single leg decline squat (SLDS) and compare patellar tendon thickness, structure, neovascularisation and symptom severity between SLDS-derived groups.
\end{abstract}

Methods $\quad 32$ male and 25 female participants attending a 5-day volleyball training camp underwent clinical evaluation by SLDS, describing the location of pain during this test using a pain map. The patellar tendon was examined using ultrasound imaging, performed by an assessor blinded to other assessments. Differences between participants experiencing local patellar tendon pain (PTP), other knee pain (OKP) or no-pain during the SLDS were evaluated.

Results $\quad$ Fifteen $(26.3 \%)$ participants experienced pain during the SLDS. Local PTP was recorded for $12.3 \%$ and OKP for $10.5 \%$ of right legs. The PTP group was distinguished from the other groups by larger thickness and cross-sectional area of the mid-patellar tendon $(\mathrm{p}<0.001)$, more frequent neovascularisation $(\mathrm{p}=0.005)$ and greater pain and disability $(\mathrm{p}<0.036)$. No differences between OKP and no-pain groups was observed.

Conclusion Adolescent non-elite volleyball players reported symptoms indicative of patellar tendinopathy. In this cohort, the SLDS test combined with a pain map was associated with imaging and questionnaire-based outcomes. 
Level of Evidence: Diagnosis, Level 2

Cross-sectional study

Keywords: ultrasound imaging, volleyball, adolescents 
Introduction

Patellar tendinopathy has a high prevalence $(44.6 \%)$ in adult volleyball athletes, ${ }^{1}$ and is the most frequent overuse injury in adolescent, elite athletes. ${ }^{2,3}$ In younger athletes, the effects of mechanical loading may compound or interact with those of maturation. ${ }^{4,5}$ These factors might expose young athletes to an increased risk of developing overuse injuries such as patellar tendinopathy. In young elite volleyball players, training volume and match exposure are predictors of the development of patellar tendinopathy, ${ }^{6}$ while other studies highlight the large ground reaction forces ( $\sim 6$ times body weight) during jumping as risk factors. ${ }^{1}$ Some studies report higher prevalence rates in men, suggesting their greater capacity to generate force may increase their risk of injury. ${ }^{1}$ Most studies have focused on adult, elite athletes, while prevalence of patellar tendinopathy in adolescent athletes, with lower training loads is understudied. Research in non-elite youth may provide insight into potential relationships between maturation, training and susceptibility to injury.

Pain on performing a task that loads the tendon was recently established by Delphi study ${ }^{7}$ as a core domain for tendinopathy. Although there is no current gold standard test, the single leg decline squat (SLDS) is proposed as a provocation test to discriminate patellar tendon pain. ${ }^{8}$ The original test was described using a $25^{\circ}$ decline board to increase load on the patellar tendon, avoiding flexion beyond $60^{\circ} .^{8}$ Variations to this test (e.g. knee flexion to only $30^{\circ}$ ) are also described. ${ }^{9}$ As the SLDS loads multiple structures in the knee, ${ }^{10}$ the location of knee pain during the clinical test might provide additional useful information. ${ }^{11}$ Localised pain over the inferior pole of the patella is described as a key diagnostic sign for patellar tendinopathy. ${ }^{12}$ However, a recent study revealed heterogeneity in pain location during the 
SLDS for elite basketballers with self-reported patellar tendinopathy, ${ }^{11}$ questioning the ability to make inferences about a pathoanatomical source of pain.

The SLDS offers an alternative to ultrasound imaging and other validated measures of the severity of tendon pain and function, such as the Victorian Institute of Sport AssessmentPatella (VISA-P). ${ }^{13}$ It can be easily administered to screen large clinical samples and is free of necessity for advanced training. However, more research is needed to inform its utility as a clinical tool for inferring patellar tendon pain (PTP).

The primary aim of this research was to determine the proportion of non-elite adolescent volleyball athletes experiencing knee symptoms, and reporting no pain, local PTP or other knee pain (OKP) areas during the SLDS test. A secondary aim was to compare ultrasound imaging characteristics (tendon thickness, structure and neovascularisation), as well as the severity of self-reported knee symptoms between no pain, local PTP and OKP groups derived from the SLDS.

Materials and methods

Participants and experimental design

Fifty-seven adolescent volleyball athletes ( 32 male, 25 female) aged 13 to 17 years attending a 5-day training program in $<<$ BLIND $>>$ agreed to participate in this cross-sectional study. The study was approved by $<<$ BLIND $>>$ University ethics committee (Approval \# 2017/896). Participants (and legal guardians) signed informed consent prior to testing and the rights of the participants were protected. All assessments were performed over a 3-day period. 
Participants completed questionnaires regarding demographics, training history and lead leg for spike jump. The Oslo Sports Trauma Research Center (OSTRC) ten item questionnaire was used to record "knee problems" (pain, ache, stiffness, swelling, instability, locking or other complaints) in one or both knees in the last week. ${ }^{14}$ Questions 1-4 were scored from 025 , with the sum of all 4 items ranging from 0 to 100; 0 represents full participation without pain or symptoms. ${ }^{14}$ The 4 -item severity score was validated in youth basketball players and found to be correlated with clinical evaluation. ${ }^{15}$ The VISA-P questionnaire was used to indicate severity of symptoms of patellar tendinopathy, with a score of 100 representing no limitations. ${ }^{13}$ Left and right limbs were scored separately for questions 1-6 of the VISA-P.

\section{Clinical evaluation}

Standing height and weight were measured, followed by assessment of the SLDS test on each leg in a random order. While balancing on a $25^{\circ}$ decline board with arms crossed and trunk vertical, participants flexed their knee to $60^{\circ}$ and rated the intensity of pain experienced on a 0-10 numerical scale. If knee pain was present, participants were asked to select the area best matching the location from one of 6 areas illustrated on a pain map (FIGURE 1). ${ }^{12}$ Three trials with 30s rest between trials were performed and an average pain rating was recorded. Test responses for each leg were classified as no-pain (pain scores in all trials=0), patellar tendon pain (PTP, average pain $>0$ and pain location 'e' FIGURE 1) or other knee pain (OKP) (average pain $>0$ and pain locations a-d or $\mathrm{f}$ ). 


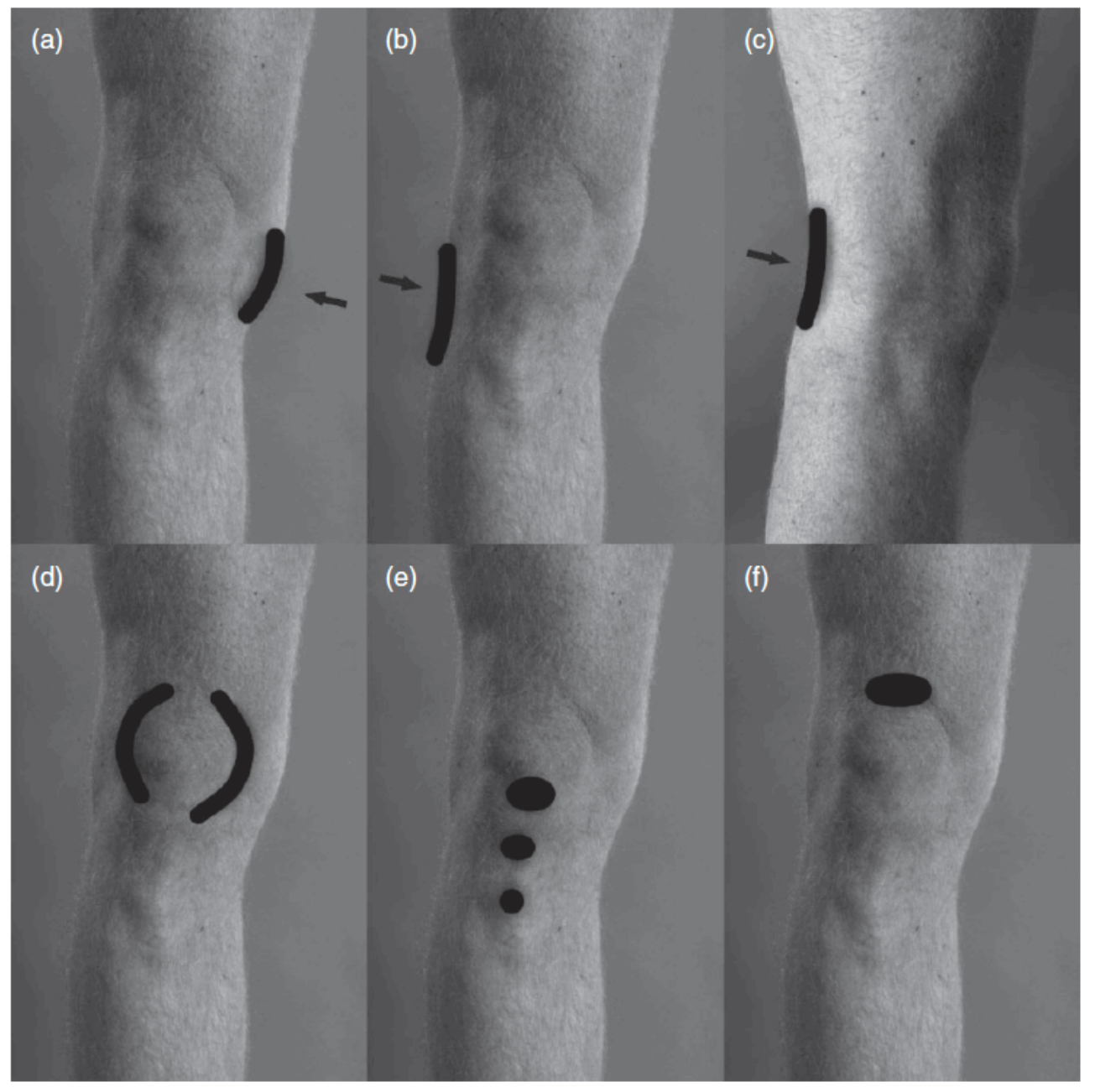

FIGURE 1: Pain map used by participants to select the picture that best matches the location of knee pain experienced during or immediately after the squat. Adapted from van der Worp et al. ${ }^{12}$

\section{Ultrasonography}

A single examiner, blinded to the participant's clinical evaluation and injury history, performed ultrasound imaging using a GE LOGIQ e with 12-4 MHz transducer (GE Healthcare, Wuxi, China). The participant was positioned in a supine lying position with the test leg (order of legs randomised) supported in $20^{\circ}$ knee flexion. The transducer was first oriented longitudinal to the mid-tendon and proximal tendon, then oriented transverse to the 
mid-tendon, recording 2 or 3 images at each location. Tendon structure was graded as 0 , homogenous echogenicity; 1, discrete hypo-echoic areas; 2, well-defined hypo-echoic areas and 3, extended hypo-echoic areas. ${ }^{16}$ Using Power Doppler, tendon neovascularity was graded as 0 , no neovessels; 1 , a few solitary blood vessels; 2, multiple vessels; 3, neovascularisation spread to whole depth of tendon. For analysis, grades 1-3 were collapsed to represent abnormal tendon structure or neovascularisation on respective scales. Tendon thickness and cross-sectional area (CSA) were measured from longitudinal and transverse images, at the thickest part of the tendon, ${ }^{16}$ using OsiriX Lite software and the average values used in analysis. Data extraction was repeated for 15 participants $(26.3 \%)$ by the same examiner. Reliability was excellent for measurement of thickness (ICC, 95\% CI: mid-tendon $0.959,0.879$ to 0.987 ; proximal tendon $0.974,0.922$ to 0.992 ; ) and good for CSA $(0.793$, 0.407 to 0.928 ), with coefficients of variation $2.8 \%, 2.5 \%$ and $8.7 \%$ and minimal detectable differences of $0.02 \mathrm{~cm}, 0.03 \mathrm{~cm}$ and $0.17 \mathrm{~cm}^{2}$ respectively. ${ }^{17}$

\section{Statistics}

Frequency and proportion of participants meeting no-pain, PTP and OKP criteria on SLDS were generated for both legs. As right and left legs are coupled and not independent, and because the prevalence of PTP was low for the left leg $(n=2)$, we performed group comparisons using data from the right leg for all participants. Normality of continuous data was confirmed using Shapiro Wilk tests and is presented as mean \pm standard deviation, with the exception of OSTRC and VISA-P questionnaires and training hours, where data did not meet normality assumptions and is presented as median [IQR]. Ultrasonography, demographic and training variables were compared between No-pain, PTP and OKP groups

using analysis of variance (ANOVA, post-hoc tests with Bonferroni correction) or $\mathrm{Chi}^{2}$ tests. Bias corrected Hedges' $g$ or odds ratios (and 95\% confidence intervals) were calculated for 
significant pairwise comparisons. ${ }^{18}$ Kruskal-Wallis ANOVA (post-hoc Wilcoxon test) were used for group comparisons of questionnaires (OSTRC and VISA-P) and training hours. Analyses were tested using Stata 13.0 and a significance level of 0.05 adopted.

Results

Fifteen $(26.3 \%)$ participants reported knee problems in the previous week using the OSTRC questionnaire. Fifteen (26.3\%) participants also experienced pain during the SLDS; $22.8 \%$ (right leg), $17.5 \%$ (left leg) and 14.0\% (bilaterally). The location of pain for right and left legs is illustrated in FIGURE 2. Pain was described as being over the patellar tendon (PTP) in $7(12.3 \%)$ of right legs and $2(4 \%)$ of left legs.
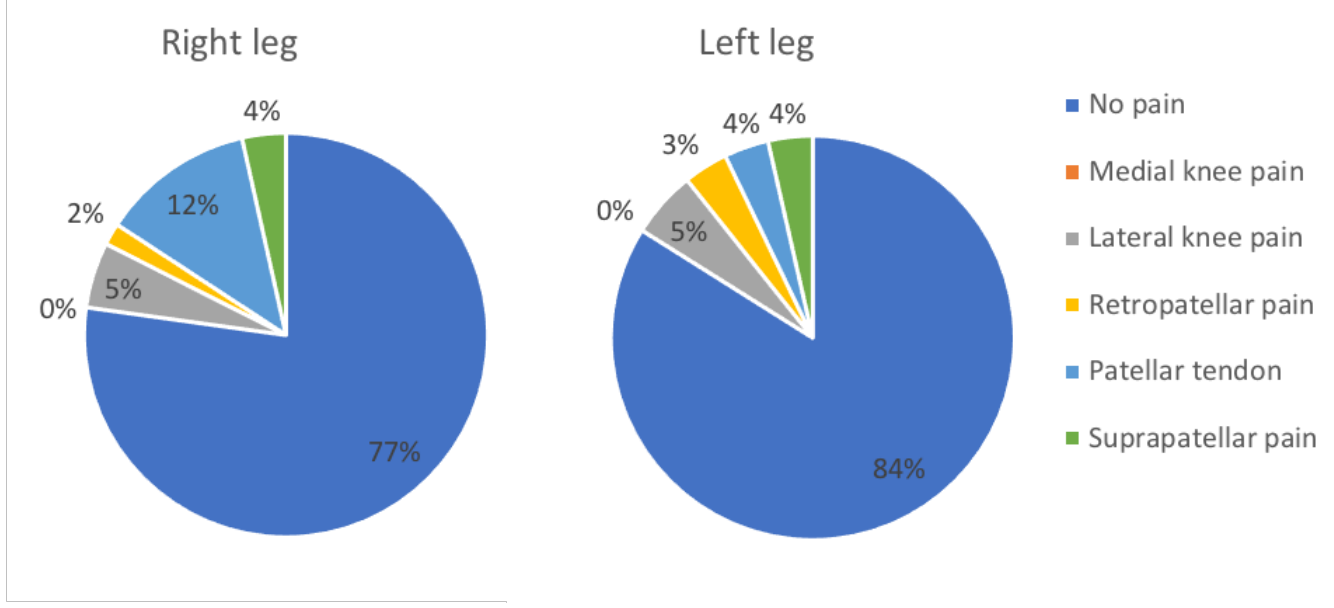

FIGURE 2: Frequency of pain in each region of the pain map for the right and left legs. No participants reported posterior knee pain.

The following group differences are based on SLDS responses for the right leg (TABLE 1). Ultrasound data for the left leg is provided for reference in TABLE 2. Comparison of Nopain, PTP and OKP groups demonstrated no significant differences in demographic, anthropometric or training variables. Average years of volleyball play was 3.0 (range 1-6) and the cohort reported a median $2 \mathrm{hrs}$ ball practice in the previous week. A similar 
proportion of preferred lead legs was observed between groups, while $95 \%$ of the cohort was right hand dominant. 
TABLE 1: Ultrasonography, demographic and training variables and symptom severity questionnaires for participants with no-pain, localised patellar tendon pain (PTP) or other knee pain (OKP) areas during the single leg decline squat test of the right limb.

$\begin{array}{lllll} & \text { No-pain } & \text { OKP } & \text { PTP } & \text { Sig. } \\ \text { n (\%) } & 44(77.2 \%) & 6(10.5 \%) & 7(12.3 \%) & \\ \text { Ultrasonography (Right leg) } & & & & \\ \text { Thickness mid-tendon }(\mathrm{cm})^{\wedge} & 0.33 \pm 0.04 & 0.31 \pm 0.02 & 0.41 \pm 0.06 & <0.001^{*} \\ \text { Thickness proximal tendon }(\mathrm{cm})^{\wedge} & 0.47 \pm 0.07 & 0.41 \pm 0.04 & 0.50 \pm 0.08 & 0.053 \\ & & & & \\ \text { CSA mid-tendon }\left(\mathrm{cm}^{2}\right)^{\wedge} & 0.86 \pm 0.14 & 0.75 \pm 0.14 & 1.07 \pm 0.14 & <0.001^{*} \\ \text { Hypo-echogenic areas } \mathrm{n}(\%) \sim & 13(29.6 \%) & 3(50 \%) & 5(71.4 \%) & 0.080 \\ & & & & \\ \text { Neovascularisation } \mathrm{n}(\%) \sim & 4(9.1 \%) & 1(16.7 \%) & 4(57.1 \%) & 0.005^{*}\end{array}$

Training variables

$\begin{array}{lllll}\text { Right lead leg during spike jump } & 13(29.6 \%) & 3(50 \%) & 3(42.9 \%) & 0.517\end{array}$

Years played volleyball^

$3.1 \pm 1.1$

$2.0 \pm 1.5$

$2.9 \pm 1.3$

0.096

Strength training (hrs)\#

$1[0,2]$

$1.5[1,2]$

$1[0,3]$

0.923

Athletic training (hrs)\#

$1.5[0.5,2.5]$

$1[1,1.5]$

$2[0,4]$

0.738

Ball practice training (hrs)\#

$2[1,3.5]$

$3[1,7]$

$4[0,7]$

0.669

Demographic variables

Male n (\%)

$24(54.6 \%) \quad 3(50 \%)$

$5(71.4 \%) \quad 0.670$

Age (years)^

$15.1 \pm 0.9$

$15.0 \pm 0.6$

$15.3 \pm 0.8$

0.827

Weight $(\mathrm{kg})^{\wedge}$

$64.5 \pm 13.9$

$66.3 \pm 15.0$

$71.7 \pm 22.8$

0.512 


$\begin{array}{lllll}\text { Height }(\mathrm{cm})^{\wedge} & 174.9 \pm 9.9 & 174.1 \pm 10.9 & 176.5 \pm 9.8 & 0.898 \\ \text { Questionnaires } & & & & \\ \text { OSTRC (0-100)\# } & 0[0,0] & 4[0,16] & 28[0,41] & 0.020^{*} \\ \text { VISA-P (0-100)\# } & 96[87,100] & 77[76,90] & 74[56,94] & 0.013^{*}\end{array}$

CSA: cross-sectional area; OSTRC: Oslo Sports Trauma Research Center; VISA-P: Victorian Institute of Sport Australia-Patellar tendon. Group differences evaluated by analysis of variance $^{\wedge}$, Chi2 or Kruskal-Wallis\# with significance indicated by asterisk.

TABLE 2: Ultrasonography data for participants with no-pain, localised patellar tendon pain (PTP) or other knee pain (OKP) areas during the single leg decline squat test of the left limb. Results were not analysed due to insufficient cases.

$\begin{array}{llll} & \text { No-pain } & \text { OKP } & \text { PTP } \\ \text { n (\%) } & 47(82.5 \%) & 8(14.0 \%) & 2(3.5 \%) \\ \text { Ultrasonography } & & & \\ \text { Thickness mid-tendon (cm) } & 0.32 \pm 0.04 & 0.32 \pm 0.04 & 0.34 \pm 0.005 \\ \text { Thickness proximal tendon (cm) } & 0.45 \pm 0.06 & 0.46 \pm 0.10 & 0.45 \pm 0.03 \\ & & & \\ \left.\text { CSA mid-tendon (cm }{ }^{2}\right) & 0.85 \pm 0.16 & 0.89 \pm 0.20 & 0.82 \pm 0.12 \\ \text { Hypo-echogenic areas n }(\%) & 12(25.5 \%) & 4(50 \%) & 0(0 \%) \\ & & & \\ \text { Neovascularisation } \mathrm{n}(\%) \sim & 6(12.8 \%) & 2(25.0 \%) & 0(0 \%)\end{array}$


Patellar tendon thickness and CSA of the mid-tendon was significantly different between groups $(\mathrm{p}<0.001)($ FIGURE 3$)$. Thickness and CSA was significantly $(\mathrm{p}<0.001)$ greater for tendons with associated PTP compared to no-pain (Hedges' $g$ : $1.86(0.96-2.71)$ and 1.48 $(0.63(0.63-2.32)$ respectively) and significantly $(\mathrm{p}<0.002)$ greater to OKP groups $(2.01$ $(0.67-3.34)$ and $2.13(0.76,3.49)$ respectively). No differences $(p>0.28)$ were found between OKP and No-pain groups. Differences in thickness at the proximal patellar tendon did not reach significance $(\mathrm{p}=0.053)$. Prevalence of abnormal tendon structure ranged between $29.6 \%$ to $71.4 \%$, but was not statistically different between groups $(p=0.08)$. Neovascularisation was significantly different between groups $(\mathrm{p}=0.005)$. A higher prevalence of neovascularisation was observed for tendons with associated PTP (57.1\%), compared to both no-pain $(9.1 \%$, odds ratio $(95 \% \mathrm{CI}): 13.3(2.2,81.9))$ and compared to OKP $(16.7 \%$; odds ratio: $6.7(0.5$, 91.3)), which were not different (TABLE 1). Neovascularisation was observed in male participants only.

VISA-P and OSTRC scores were significantly different between groups $(p=0.004$ and 0.036 respectively), with more severe symptoms for participants reporting PTP (TABLE 1). 


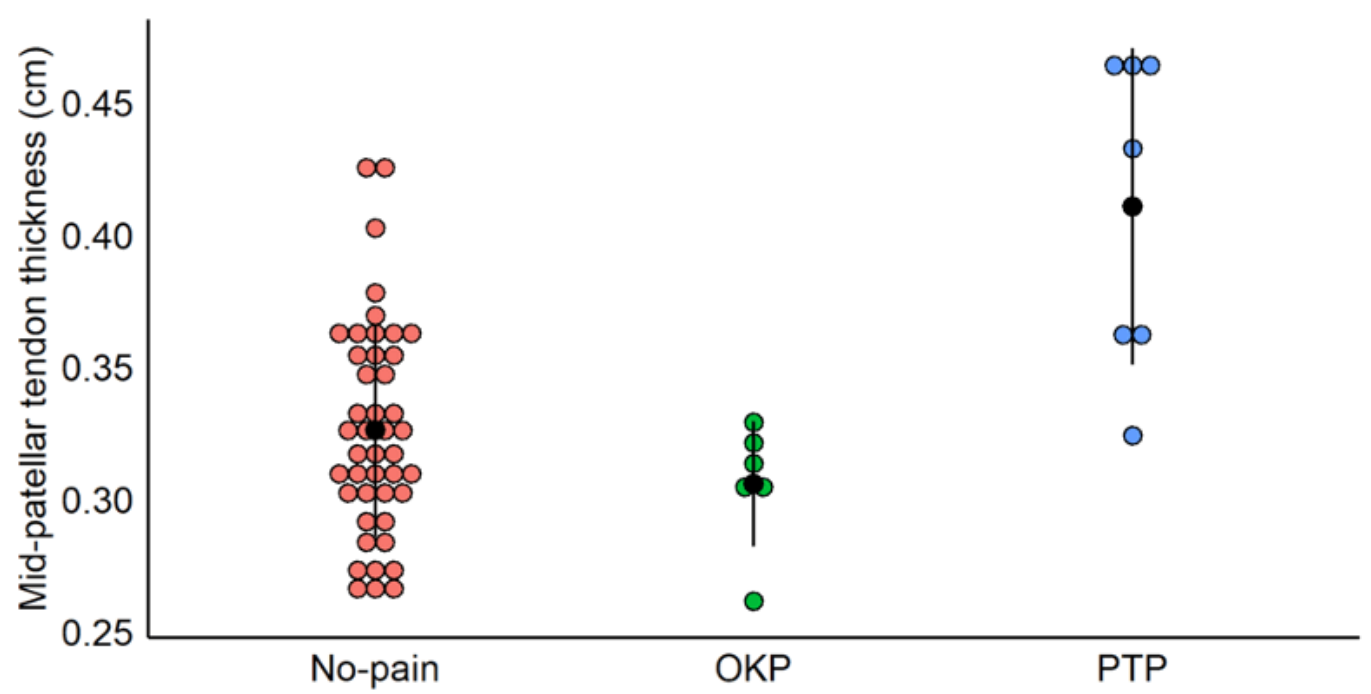

FIGURE 3: Mid-tendon thickness of the patellar tendon for participants with no-pain, localised patellar tendon pain (PTP) or other knee pain (OKP) areas during the single leg decline squat test of the right limb.

\section{Discussion}

Approximately 1 in 4 non-elite volleyball athletes aged 13-17 years reported knee symptoms in the previous week and a similar proportion experienced pain during a SLDS. While these estimates are lower than those of a recent study of elite, adult basketball athletes, where 1 in 2 experienced a positive SLDS test, ${ }^{11}$ they suggest that knee pain is a common problem in volleyball players of all ages and training background. When the right leg was considered, local pain over the patellar tendon was the most common area when symptoms during the test were reported (12.3\%). VISA-P and OSTRC scores and some ultrasonography variables were also different between participants with local PTP compared to participants with other areas of pain. 
The results demonstrated significantly greater tendon dimensions at the mid-tendon in the PTP group compared to both OKP and no-pain groups. As no differences in demographic, anthropometric or training variables were found between groups, we are confident that differences in tendon dimensions are not confounded by maturation or recent mechanical loading. Body mass and muscle mass increase substantially from childhood to adulthood, requiring the weight-bearing tendons to tolerate higher loads. ${ }^{4}$ This maturational process includes dimensional growth as well as changes in the tendon's intrinsic material properties, such as by increased collagen density. Similar changes are proposed to occur following resistance training in adults, although increases in tendon thickness were not observed in adolescent athletes despite high training volume over a 1.7 year period. ${ }^{19}$ Contrary to other studies in elite athletes, ${ }^{1,3}$ we did not find a higher prevalence of patellar tendinopathy in males, despite tendon thickness being greater in males (mid-tendon $0.35 \pm 0.05$ and $0.31 \pm$ $0.04 \mathrm{~cm}$ respectively, $\mathrm{p}<0.001)$.

A systematic review of tendinopathy in children and adolescents found studies using clinical examination typically reported lower prevalence rates (2-18\%) of tendinopathy than studies using ultrasonography $(20-38 \%) .{ }^{20}$ This is consistent with findings that asymptomatic pathology (e.g. presence of hypoechoic areas or neovascularisation) exists in a large proportion of the active adult population..$^{2}$ In our cohort, the proportion of participants with structural tendon abnormality was not statistically different between No-pain, PTP and OKP groups. In contrast, neovascularisation was significantly more likely in participants with PTP than both other groups. These findings suggest assessment using Doppler ultrasound examination provides additional information to grey-scale imaging alone when evaluating the adolescent patellar tendon. As previous studies demonstrate intratendinous flow can increase 
following exercise, ${ }^{21}$ a lack of standardisation of load immediately prior to imaging may have influenced findings.

As patellar tendinopathy begins in childhood and increases in prevalence with age up to 18 years, ${ }^{20}$ and is difficult to treat, early screening to identify at-risk individuals is needed. The current results support the use of the SLDS test in evaluating patellar tendinopathy when information about the location of symptoms during testing is taken into account. This study is not without limitations. First, the small sample of symptomatic tendons, particularly on the left side will affect robustness of these conclusions. For this reason, evaluation of the diagnostic accuracy of this assessment to identify PTP was not conducted. Second, our physical examination of participants was limited to performance of the SLDS test and not a full clinical examination. Other clinical information is likely to increase the likelihood of a diagnosis of patellar tendinopathy and assist in identification of modifiable factors. ${ }^{22} \mathrm{~A}$ previous study of adult athletes indicated improved diagnostic accuracy for diagnosing patellar tendinopathy when multiple tests were used, compared to that of isolated tests. ${ }^{9}$

\section{Conclusion}

In a cohort of non-elite volleyball players aged 13-17years, individuals reporting localised pain over the patellar tendon during the SLDS test were different from participants without pain or other areas of knee pain based on patellar tendon dimensions, neovascularisation and VISA-P scores. Use of a pain map is proposed to be a critical component of the test. These results support the use of the SLDS test for early screening during the adolescent years, when symptoms of patellar tendinopathy may arise. 


\section{Highlights}

- Pain during the SLDS test is common in non-elite adolescent volleyball athletes.

- Local patellar tendon pain was associated with increased mid-tendon thickness and cross-sectional area, neovascularisation and VISA-P scores.

- Clinicians should utilise a pain map in conjunction with the SLDS for evaluation of patellar tendinopathy. 
References

1. Lian, O.B., L. Engebretsen, and R. Bahr. Prevalence of jumper's knee among elite athletes from different sports: a cross-sectional study Am J Sports Med. 2005; 33:4: 561-7.http://dx.doi.org/10.1177/0363546504270454

2. Gisslen, K., C. Gyulai, K. Soderman, and H. Alfredson. High prevalence of jumper's knee and sonographic changes in Swedish elite junior volleyball players compared to matched controls Br J Sports Med. 2005; 39:5: 298-

301.http://dx.doi.org/10.1136/bjsm.2004.014290

3. Cassel, M., H. Baur, A. Hirschmuller, A. Carlsohn, K. Frohlich, and F. Mayer. Prevalence of Achilles and patellar tendinopathy and their association to intratendinous changes in adolescent athletes Scand J Med Sci Sports. 2015; 25:3: e310-8.http://dx.doi.org/10.1111/sms. 12318

4. Waugh, C.M., A.J. Blazevich, F. Fath, and T. Korff. Age-related changes in mechanical properties of the Achilles tendon J Anat. 2012; 220:2: 14455.http://dx.doi.org/10.1111/j.1469-7580.2011.01461.x

5. Mersmann, F., G. Charcharis, S. Bohm, and A. Arampatzis. Muscle and Tendon Adaptation in Adolescence: Elite Volleyball Athletes Compared to Untrained Boys and Girls Front Physiol. 2017; 8:417.http://dx.doi.org/10.3389/fphys.2017.00417

6. Visnes, H. and R. Bahr. Training volume and body composition as risk factors for developing jumper's knee among young elite volleyball players Scand J Med Sci Sports. 2013; 23:5: 607-13.http://dx.doi.org/10.1111/j.1600-0838.2011.01430.x

7. Vicenzino, B., R.J. de Vos, H. Alfredsonet al. ICON 2019-International Scientific Tendinopathy Symposium Consensus: There are nine core health-related domains for tendinopathy (CORE DOMAINS): Delphi study of healthcare professionals and patients Br J Sports Med. 2020; 54:8: 444-451.http://dx.doi.org/10.1136/bjsports2019-100894

8. Purdam, C. Discriminative ability of functional loading tests for adolescent jumper's knee Physical Therapy in Sport. 2003; 4:3-9.

9. Mendonca Lde, M., J.M. Ocarino, N.F. Bittencourt, L.M. Fernandes, E. Verhagen, and S.T. Fonseca. The Accuracy of the VISA-P Questionnaire, Single-Leg Decline Squat, and Tendon Pain History to Identify Patellar Tendon Abnormalities in Adult Athletes J Orthop Sports Phys Ther. 2016; 46:8: 673-

80.http://dx.doi.org/10.2519/jospt.2016.6192

10. Zwerver, J., S.W. Bredeweg, and A.L. Hof. Biomechanical analysis of the single-leg decline squat Br J Sports Med. 2007; 41:4: 264-

8.http://dx.doi.org/10.1136/bjsm.2006.032482

11. Hannington, M., S. Docking, J. Cook, S. Edwards, and E. Rio. Self-reported jumpers' knee is common in elite basketball athletes - But is it all patellar tendinopathy? Phys Ther Sport. 2020; 43:58-64.http://dx.doi.org/10.1016/j.ptsp.2020.01.012

12. van der Worp, H., M. van Ark, J. Zwerver, and I. van den Akker-Scheek. Risk factors for patellar tendinopathy in basketball and volleyball players: a cross-sectional study Scand J Med Sci Sports. 2012; 22:6: 783-90.http://dx.doi.org/10.1111/j.1600$\underline{0838.2011 .01308 . \mathrm{x}}$

13. Visentini, P.J., K.M. Khan, J.L. Cook, Z.S. Kiss, P.R. Harcourt, and J.D. Wark. The VISA score: an index of severity of symptoms in patients with jumper's knee (patellar tendinosis). Victorian Institute of Sport Tendon Study Group J Sci Med Sport. 1998; $1: 1: 22-8$.

14. Clarsen, B., G. Myklebust, and R. Bahr. Development and validation of a new method for the registration of overuse injuries in sports injury epidemiology: the Oslo Sports 
Trauma Research Centre (OSTRC) overuse injury questionnaire Br J Sports Med. 2013; 47:8: 495-502.http://dx.doi.org/10.1136/bjsports-2012-091524

15. Owoeye, O.B.A., J.P. Wiley, R.E.A. Walker, L. Palacios-Derflingher, and C.A. Emery. Diagnostic Accuracy of a Self-report Measure of Patellar Tendinopathy in Youth Basketball J Orthop Sports Phys Ther. 2018; 48:10: 758-

766.http://dx.doi.org/10.2519/jospt.2018.8088

16. Sunding, K., M. Fahlstrom, S. Werner, M. Forssblad, and L. Willberg. Evaluation of Achilles and patellar tendinopathy with greyscale ultrasound and colour Doppler: using a four-grade scale Knee Surg Sports Traumatol Arthrosc. 2016; 24:6: 198896.http://dx.doi.org/10.1007/s00167-014-3270-4

17. Weir, J.P. Quantifying test-retest reliability using the intraclass correlation coefficient and the SEM $J$ Strength Cond Res. 2005; 19:1: 231-

40.http://dx.doi.org/10.1519/15184.1

18. Monitoring, C.C.f.E. Effect size calculator. 2020; Available from: https://www.cem.org/effect-size-calculator.

19. Visnes, H., A. Tegnander, and R. Bahr. Ultrasound characteristics of the patellar and quadriceps tendons among young elite athletes Scand J Med Sci Sports. 2015; 25:2: 205-15.http://dx.doi.org/10.1111/sms.12191

20. Simpson, M., E. Rio, and J. Cook. At What Age Do Children and Adolescents Develop Lower Limb Tendon Pathology or Tendinopathy? A Systematic Review and Meta-analysis Sports Med. 2016; 46:4: 545-57.http://dx.doi.org/10.1007/s40279-015$\underline{\text { 0438-0 }}$

21. Cook, J.L., Z.S. Kiss, R. Ptasznik, and P. Malliaras. Is vascularity more evident after exercise? Implications for tendon imaging AJR Am J Roentgenol. 2005; 185:5: 113840.http://dx.doi.org/10.2214/AJR.04.1205

22. Mendonca, L.D., J.M. Ocarino, N.F.N. Bittencourt, L.G. Macedo, and S.T. Fonseca. Association of Hip and Foot Factors With Patellar Tendinopathy (Jumper's Knee) in Athletes J Orthop Sports Phys Ther. 2018; 48:9: 676-

684.http://dx.doi.org/10.2519/jospt.2018.7426 